\title{
Study of the structure of the QCD vacuum with valence overlap fermions and monopoles.
}

\author{
Toru Sekido* ${ }^{* a, b}$, Katsuya Ishiguro ${ }^{a, b}$, Yoshiaki Koma ${ }^{c}$, Yoshifumi Nakamura ${ }^{d}$, \\ Gerrit Schierholz ${ }^{d, e}$ and Tsuneo Suzuki ${ }^{a, b}$ \\ ${ }^{a}$ Institute for Theoretical Physics, Kanazawa University,Kanazawa 920-1192, Japan \\ ${ }^{b}$ RIKEN, Radiation Laboratory, Wako 351-0158, Japan \\ ${ }^{c}$ Numazu College of Technology, Numazu 410-8501, Japan \\ ${ }^{d}$ John von Neumann Institute NIC/DESY Zeuthen, 15738 Zeuthen, Germany \\ ${ }^{e}$ Deutsches Elektronen-Synchrotron DESY, 22603 Hamburg, Germany \\ E-mail: toryu@hep.s.kanazawa-u.ac.jp
}

\begin{abstract}
Overlap fermions which have an exact lattice chiral symmetry are used for investigating the topological structure of the QCD vacuum. We study the relation between confinement and chiral properties by focusing on monopoles in the Maximally-Abelian gauge at finite temperature. We calculate the eigenvalue of the overlap Dirac operator on Abelian, monopole and photon backgrounds. We find that the spectral densities on the Abelian and the monopole backgrounds have a gap only in the deconfinement phase. This behaviors are similar to that on the non-Abelian background. However on the photon background, there is a gap even in the confinement phase. Our preliminary results suggest that the monopoles responsible for confinement play an important role also for the chiral phase transition.
\end{abstract}

The XXV International Symposium on Lattice Field Theory

July 30-4 August 2007

Regensburg, Germany

\footnotetext{
* Speaker.
} 


\section{Introduction}

The understanding of the vacuum structure in QCD is an important research theme of theoretical particle physics. In particular quark confinement and spontaneous chiral symmetry breaking are interesting phenomena.

Confinement is believed to be explained by the dual Meissner effect [1,2]. This is an analogy of the superconductor. In this scheme magnetic monopoles play a very important role like Cooper pairs in the superconductor. To extract magnetic monopoles, we usually need some partial gauge fixing keeping a residual Abelian symmetry. Numerically when we perform the MaximallyAbelian (MA) projection [3,4], we easily get interesting results supporting the dual Meissner effect. Very recently it is shown that similar results supporting the dual Meissner effect are obtained even without any gauge-fixing [5] when one performs very precise numerical simulations.

With respect to the relation between confinement and chiral symmetry breaking, Miyamura has shown that the monopoles in MA gauge are responsible for chiral condensate using $S U(2)$ staggered fermions [6].

It is desirable to study the relation using a $S U(3)$ fermion action having a better chiral symmetry. Overlap fermions have an exact chiral symmetry on the lattice at a finite lattice spacing $a$ [7]. Their implementation of the chiral symmetry and the possibility to exactly define the index theorem on the lattice allow us to investigate the relationship between topological properties of gauge fields like monopoles and chiral properties of fermions. Here we adopt magnetic monopoles in the MA gauge.

\section{Abelian projection}

First we implement the MA gauge $[8,9,10]$ and extract Abelian link fields $\left(\theta_{\mu}^{a}(s)\right)$ from the gauge-fixed non-Abelian ones. Then we define an Abelian field strength:

$$
\theta_{\mu \nu}^{a}(s)=\partial_{\mu} \theta_{v}^{a}(s)-\partial_{v} \theta_{\mu}^{a}(s)=\bar{\theta}_{\mu v}^{a}(s)+2 \pi n_{\mu \nu}^{a}(s),
$$

where

$$
\sum_{a} \bar{\theta}_{\mu v}^{a}(s)=\sum_{a} n_{\mu \nu}^{a}(s)=0
$$

are satisfied[8]. Here $\bar{\theta}_{\mu \nu}^{a}$ is interpreted as an electromagnetic flux through a plaquette, and $n_{\mu \nu}^{a}$ corresponds to the number of the Dirac strings piercing the plaquette. The monopole current is defined as

$$
\begin{gathered}
k_{\mu}^{a}(s)=\frac{1}{4 \pi} \varepsilon_{\mu \nu \rho \sigma} \partial_{\nu} \bar{\theta}_{\rho \sigma}^{a}(s+\hat{\mu})=-\frac{1}{2} \varepsilon_{\mu \nu \rho \sigma} \partial_{\nu} n_{\rho \sigma}^{a}(s+\hat{\mu}), \\
\sum_{a} k_{\mu}^{a}(s)=0, \quad \partial_{\mu}^{\prime} k_{\mu}^{a}(s)=0 .
\end{gathered}
$$

An Abelian link field is decomposed into the monopole part and the photon part using the Hodge decomposition:

$$
\begin{aligned}
\theta_{\mu}^{a}(s) & =-\sum_{s^{\prime}} D\left(s-s^{\prime}\right)\left[\partial_{v}^{\prime} \theta_{v \mu}^{a}\left(s^{\prime}\right)+\partial_{\mu}\left(\partial_{v}^{\prime} \theta_{v}^{a}\left(s^{\prime}\right)\right)\right] \\
& =\theta_{m o n, \mu}^{a}(s)+\theta_{p h, \mu}^{a}(s),
\end{aligned}
$$




$$
\begin{aligned}
\theta_{m o n, \mu}^{a}(s) & =-2 \pi \sum_{s^{\prime}} D\left(s-s^{\prime}\right) \partial_{v}^{\prime} n_{v \mu}^{a}\left(s^{\prime}\right), \\
\theta_{p h, \mu}^{a}(s) & =-\sum_{s^{\prime}} D\left(s-s^{\prime}\right)\left[\partial_{v}^{\prime} \bar{\theta}_{v \mu}^{a}\left(s^{\prime}\right)+\partial_{\mu}\left(\partial_{v}^{\prime} \theta_{v}^{a}\left(s^{\prime}\right)\right)\right],
\end{aligned}
$$

where $D\left(s-s^{\prime}\right)$ is the lattice Coulomb propagator. Monopole and photon link fields are defined as

$$
U_{m o n, \mu}(s)=\left(\begin{array}{ccc}
e^{i \theta_{m o n, \mu}^{1}(s)} & 0 & 0 \\
0 & e^{i \theta_{\text {mon }, \mu}^{2}(s)} & 0 \\
0 & 0 & e^{i \theta_{\text {mon }, \mu}^{3}(s)}
\end{array}\right), U_{p h, \mu}(s)=\left(\begin{array}{ccc}
e^{i \theta_{p h, \mu}^{1}(s)} & 0 & 0 \\
0 & e^{i \theta_{p h, \mu}^{2}(s)} & 0 \\
0 & 0 & e^{i \theta_{p h, \mu}^{3}(s)}
\end{array}\right) .
$$

\section{Overlap fermion}

The overlap operator is defined by

$$
\begin{aligned}
D_{N} & =\frac{\rho}{a}\left(1+\frac{X}{\sqrt{X^{\dagger} X}}\right)=\frac{\rho}{a}(1+\operatorname{sgn}(X))=\frac{\rho}{a}\left(1+\gamma_{5} \operatorname{sgn}(H)\right), \\
X & =D_{W}-\frac{\rho}{a}, \quad H=\gamma_{5} X,
\end{aligned}
$$

where $D_{W}$ is the Wilson Dirac operator. To compute this sign function $\operatorname{sgn}(X)$ we use the minmax polynomial approximation. We compute the $\mathscr{O}(40)$ lowest eigenvalues of $H$ and treat the sign function on the corresponding subspace exactly.

We study properties of the low-lying modes of the overlap Dirac operator.

$$
D_{N} \psi_{n}=\lambda_{n} \psi_{n},
$$

where $\psi_{n}$ is an eigenstate and $\lambda_{n}$ is the eigenvalue. Low-lying modes are important in understanding topological properties [11]. The difference between the number of zero modes with positive and negative chirality is related to the topological charge. And near-zero modes determine the value of the quark condensate by the Banks-Casher relation [12].

\section{Numerical results}

First we carry out simulations of quenched QCD on $N_{s}^{3} \times N_{t}=16^{3} \times 4$ lattice in the range of $T / T_{c}=0.6 \sim 2$ (Table. 1). We employ the Wilson gauge action.

The spontaneous breaking of chiral symmetry by the dynamical creation of a nonvanishing chiral condensate $\langle\bar{\psi} \psi\rangle$ is related to the spectral density $\rho(\lambda)$ of the Dirac operator near zero mode by the Banks-Casher relation $\langle\bar{\psi} \psi\rangle=-\frac{\pi}{V} \rho(0)$. We compute the 50 lowest eigenvalues on

\begin{tabular}{|c|c|c|c|c|c|}
\hline$\beta$ & 5.50 & 5.55 & 5.80 & 5.90 & 6.0 \\
\hline$a^{-1}[\mathrm{MeV}] \sim$ & 692.5 & 795.7 & 1446.4 & 1766.6 & 2118.4 \\
\hline$T / T_{c} \sim$ & 0.6 & 0.75 & 1.3 & 1.5 & 2 \\
\hline
\end{tabular}

Table 1: The table of beta, lattice spacing and $T / T_{c}[13,14]$. 


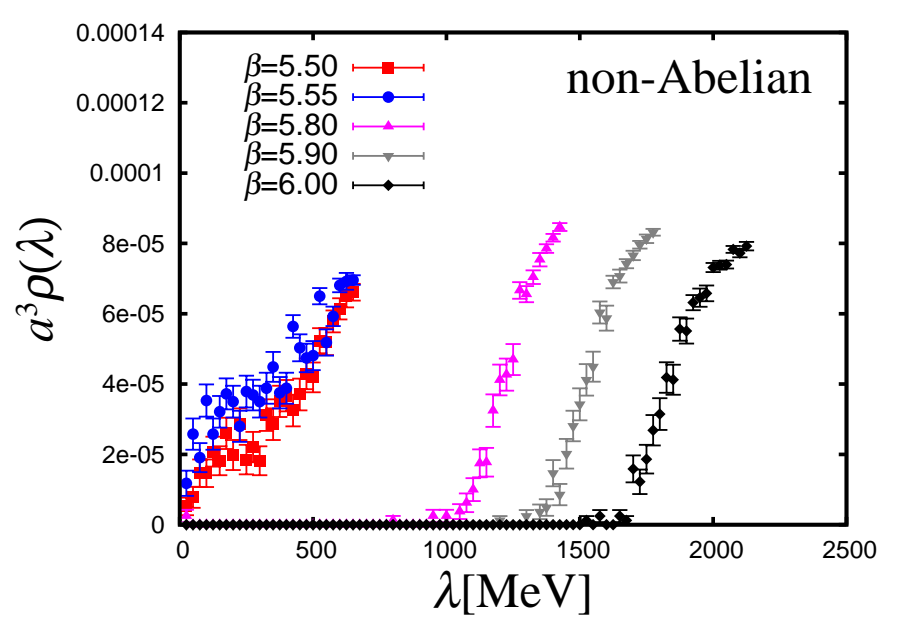

Figure 1: The spectral density of the overlap operator in the vicinity of the phase transition. The bin size of this spectral density is $25[\mathrm{MeV}]$.

every configuration by using the Arnoldi-algorithm. In our spectral analysis we use the improved massless Neuberger operator [15]

$$
D^{\mathrm{imp}}=\left(1-\frac{a}{2 \rho} D_{N}\right)^{-1} D_{N}
$$

The resulting eigenvalues $\lambda_{\text {imp }}$ of $D^{\text {imp }}$ are the stereographical projection of the eigenvalues $\lambda_{N}$ of $D_{N}$ from the Ginsparg-Wilson circle to the imaginary axis. The non-zero eigenvalues appear in pairs $\lambda_{\text {imp }}= \pm i \lambda$. In terms of these imaginary parts $\lambda$, the spectral density and the characteristic properties of the eigenmodes are discussed. In Fig. 1 we plot the spectral density. One can clearly
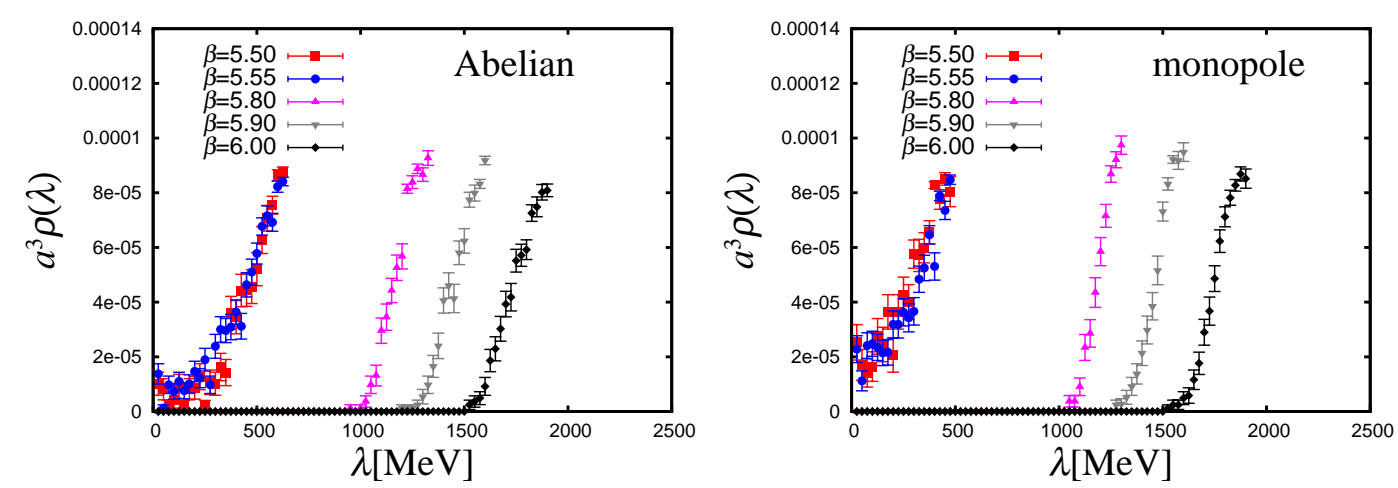

Figure 2: The spectral density of the overlap operator in the vicinity of the phase transition on the Abelian(left) and the monopole(right) backgrounds. 


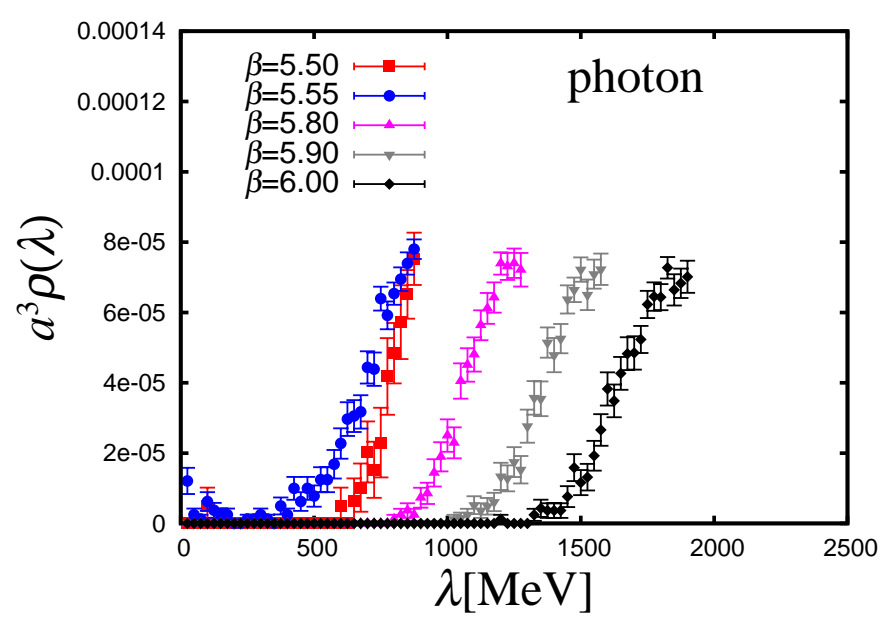

Figure 3: The spectral density of the overlap operator in the vicinity of the phase transition on the photon background.
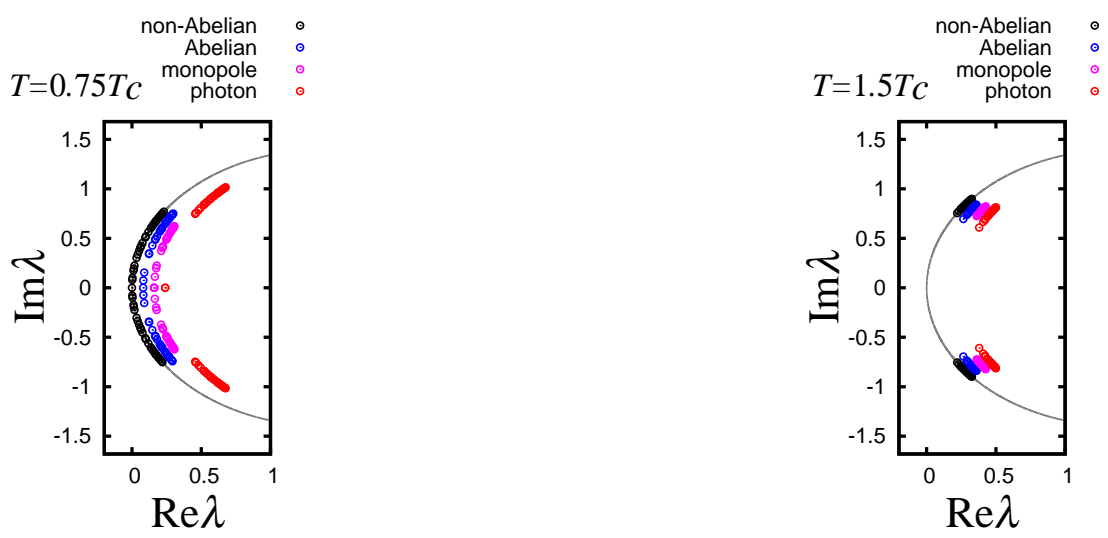

Figure 4: Two typical spectra. The left figure is in the confinement phase. The right one is in the deconfinement phase. The full curve is the Ginsparg-Wilson circle. In this figure we plot the original eigenvalue of the overlap Dirac operator. On each background we shifted these data points to see easily.

see nonvanishing chiral condensate in the confinement phase at $\beta=5.55$ below the phase transition temperature and a large gap in the spectrum at $\beta=5.8$ in the deconfinement phase.

In Fig. 2 we plot the spectral density on the Abelian and the monopole backgrounds. It seems that these behaviors are almost the same as the spectral density calculated using the non-Abelian link fields. These figures suggest the existence of a nonvanishing chiral condensate in the confinement phase and there is a gap for each $\beta$ in the spectrum in the deconfinement phase. In Fig. 3 we plot the spectral density on the photon background. On the photon background we see a gap for all $\beta$ 's. It seems that the photon part is irrelevant for chiral symmetry breaking. These results are very interesting since monopoles known as a key quantity in the confinement mechanism through the dual Meissner effect are important also for chiral symmetry breaking.

In Fig. 4 we show the typical spectra in both phases. We clearly see a gap on all backgrounds 
in the deconfinement phase and no gap in the confinement phase except on the photon background.

Finally we find that on the Abelian, the monopole and the photon backgrounds, distribution of zero modes are different from that on the non-Abelian background. On the Abelian, the monopole and the photon backgrounds, we obtain such results that the number of the zero modes is not always equal to the absolute value of the topological charge, and both $n_{+}$(the number of the zero modes with the positive chirality) and $n_{-}$(the number of the zero modes with the negative chirality) are finite in some configurations. On the other hand, it is numerically known that whether $n_{+}$or $n_{-}$ can be non-zero on the non-Abelian background. Hence the topological charge is equal to $n_{+}$or $n_{-}$in the non-Abelian case.

\section{Summary}

Overlap fermions which have an exact lattice chiral symmetry are used for investigating the topological structure of the QCD vacuum. We have studied the relation between confinement and chiral properties by focusing on monopoles in the Maximally-Abelian gauge at finite temperature. We calculated the eigenvalue of the overlap Dirac operator on Abelian, monopole and photon backgrounds. We found that the spectral densities on the Abelian and monopole backgrounds have a gap only in the deconfinement phase. The behaviors are similar to that on the non-Abelian background. However on the photon background, there is a gap even in the confinement phase. Our preliminary results suggest that the monopoles responsible for confinement play an important role also for the chiral phase transition.

Acknowledgements: One of the authors (T. Sekido) is grateful to M.I. Polikarpov, S.M. Morozov for the discussions on numerical codes. The numerical simulations of this work were done using RSCC computer clusters in RIKEN and SX8 at RCNP of Osaka University and SX8 at YITP in Kyoto University. The authors would like to thank RIKEN, RCNP and YITP for their support of computer facilities.

\section{References}

[1] G. 't Hooft, in Proceedings of the EPS International, edited by A. Zichichi, p. 1225, 1976.

[2] S. Mandelstam, Phys. Rept. 23, 245 (1976).

[3] T. Suzuki, Nucl. Phys. Proc. Suppl. 30, 176 (1993).

[4] Y. Koma, M. Koma, E.-M. Ilgenfritz, T. Suzuki, and M. I. Polikarpov, Phys. Rev. D68, 094018 (2003).

[5] T. Suzuki, K. Ishiguro, Y. Koma and T. Sekido, arXiv:0706.4366(2007).

[6] O. Miyamura, Phys. Lett. B 353, 91 (1995).

[7] H. Neuberger, Phys. Lett. B 417, 141 (1998).

[8] F. Brandstater, U. J. Wiese and G. Schierholz, Phys. Lett. B 272, 319 (1991).

[9] T. Suzuki, Prog. Theor. Phys. 69, 1827 (1983).

[10] A. S. Kronfeld, M. L. Laursen, G. Schierholz, and U. J. Wiese, Phys. Lett. B198, 516 (1987). 
[11] E. M. Ilgenfritz, K. Koller, Y. Koma, G. Schierholz, T. Streuer and V. Weinberg, Phys. Rev. D 76, 034506 (2007).

[12] T. Banks and A. Casher, Nucl. Phys. B 169, 103 (1980).

[13] S. Necco and R. Sommer, Nucl. Phys. B 622, 328 (2002).

[14] J. Fingberg, U. M. Heller and F. Karsch, Nucl. Phys. B 392, 493 (1993).

[15] T. W. Chiu, C. W. Wang and S. V. Zenkin, Phys. Lett. B 438, 321 (1998). 